\title{
Estimating 3D Strain from 4D Cine-MRI and Echocardiography: In-Vivo Validation
}

\author{
Xenophon Papademetris ${ }^{1}$, Albert J. Sinusas ${ }^{2,3}$, Donald P. Dione ${ }^{2}$, \\ R. Todd Constable ${ }^{2}$, and James S. Duncan ${ }^{1,3}$ \\ 1 Departments of Electrical Engineering, \\ 2 Diagnostic Radiology, \\ 3 Medicine, \\ Yale University New Haven, CT 06520-8042 \\ papad@noodle.med.yale.edu
}

\begin{abstract}
The quantitative estimation of regional cardiac deformation from 3D image sequences has important clinical implications for the assessment of myocardial viability. The validation of such image-derived estimates, however, is a non-trivial problem as it is very difficult to obtain ground truth. In this work we present an approach to validating strain estimates derived from 3D cine-Magnetic Resonance (MR) and 3D Echocardiography (3DE) images using our previously-developed shape-based tracking algorithm. The images are segmented interactively and then initial correspondence is established using a shape-tracking approach. A dense motion field is then estimated using a transversely linear elastic model, which accounts for the fiber directions in the left ventricle. The dense motion field is in turn used to calculate the deformation of the heart wall in terms of strains. The strains obtained using our algorithm are compared to strains estimated using implanted markers and sonomicrometers, which are used as the gold standards. These preliminary studies show encouraging results.
\end{abstract}

\section{Introduction}

It is the fundamental goal of many forms of cardiac imaging and image analysis to measure the regional function of the left ventricle (LV) in an effort to isolate the location and extent of ischemic or infarcted myocardium. In addition, the current management of acute ischemic heart disease is directed at establishing coronary reperfusion and, in turn, myocardial salvage. Regional function may also be related to the degree of salvage achieved.

However, while there have been many methods proposed for the estimation of 3D left ventricular deformation (e.g. $[1,6,7,16]$ ), the validation of these results is an extremely important and often neglected aspect of work in this area. Often phantoms are used with known shapes and displacements usable as ground truth (e.g. Kraitchman[8]). In Young [19] it was shown that away from the free surfaces of the gel-phantom, a Rivlin-Mooney [9] analytic model accurately reproduced the 2-D displacement of magnetic tags. This showed agreement between the 
theory (model) and the image-derived displacements. However, in vivo measurements of the beating heart usually present additional complexities. An alternative validation method is to use simulated images with known ground truth (e.g. Amini[1], Prince [14] and Haber [6]). One example[1] uses a kinematic model of the left ventricular motion by Arts [2] within an MR tag image simulator [18] to generate synthetic images with known displacements. In the shape-tracking work of Shi[17], implanted markers are used as the gold standard. These markers are physically implanted on the myocardium before the imaging. Here, algorithm generated displacements are compared to the marker-displacements (these are easily identifiable from the images). This technique has the disadvantage of comparing trajectories for a small number of points, however, it is done on real data as opposed to simulations. However, in all previous efforts including those noted above, to the best of our knowledge, there has been no validation of estimates of in-vivo cardiac deformation (Shi et al[17] only validate displacements.)

In this paper we describe the experiments used to validate strains estimates derived from our algorithm, which uses a transversely isotropic model for the left ventricle and shape-based displacements [12]. We first briefly review the algorithm used to estimate the deformation and then describe the methodology used for the validation. We then present results for the validation of these deformation estimates derived from Magnetic Resonance (MR) and 3D-Echocardiography (3DE) image sequences.

\section{Our Algorithm}

\subsection{Image Acquisition}

Canine MR-images: ECG-gated magnetic resonance imaging was performed on a GE Signa 1.5 Tesla scanner. Axial images through the LV were obtained with the gradient echo cine technique. The imaging parameters were: section thickness $=5$ $\mathrm{mm}$, no intersection gap, $40 \mathrm{~cm}$ field of view, TE $13 \mathrm{msec}$, TR $28 \mathrm{msec}$, flip angle 30 degrees, flow compensation in the slice and read gradient directions, $256 \times$ 128 matrix and 2 excitations. The resulting 3D image set consists of sixteen $2 \mathrm{D}$ image slices per temporal frame, and sixteen temporal 3D frames per cardiac cycle, with an in-plane resolution of $1.6 \mathrm{~mm}$ and a slice thickness of $5 \mathrm{~mm}$.

$3 D$ Echocardiography (3DE): The 3DE images were acquired using an HP Sonos 5500 Ultrasound System with a 3D transducer (Transthoracic OmniPlane 21349A (R5012)). The 3D-probe was placed at the apex of the left ventricle of an openchest dog using a small ultrasound gelpad (Aquaflex) as a standoff [12]. Each acquisition consisted of 13-17 frames per cardiac cycle depending on the heart rate. The angular slice spacing was 5 degrees resulting in 36 image slices for each $3 \mathrm{D}$ frame.

\subsection{Segmentation and Shape-Based Tracking}

The endocardial and epicardial surfaces were extracted interactively[11] and then sampled to 0.5 voxel resolution. Next, curvatures are calculated and the shape 
based tracking algorithm applied to generate a set of initial matches and confidence measures for all the points on the surface. Given a set of displacement vector measurements $u^{m}$ and confidence measures $c^{m}$ we model theses estimates probabilistically by assuming that the noise in the individual measurements as normally distributed with zero mean and a variance $\sigma^{2}$ equal to $\frac{1}{c^{m}}$. These assumptions result in a measurement probability of the form:

$$
p\left(u^{m} \mid u\right)=\frac{1}{\sqrt{2 \pi \sigma^{2}}} e^{-\frac{\left(u-u^{m}\right)^{2}}{2 \sigma^{2}}}
$$

\subsection{Modeling the Myocardium}

The left ventricular myocardium is modeled using a biomechanical model. We use a transversely linear elastic model which allows us to incorporate information about the preferential stiffness of the tissue along fiber directions from [5]. These fiber directions are shown in figure 1. The model is described in terms of an internal or strain energy function of the form:

$$
W=\epsilon^{\prime} C \epsilon
$$

where $\epsilon$ is the strain and $C$ is the $6 \times 6$ matrix containing the elastic constants which define the material properties (see $[12,13]$ for the details.)
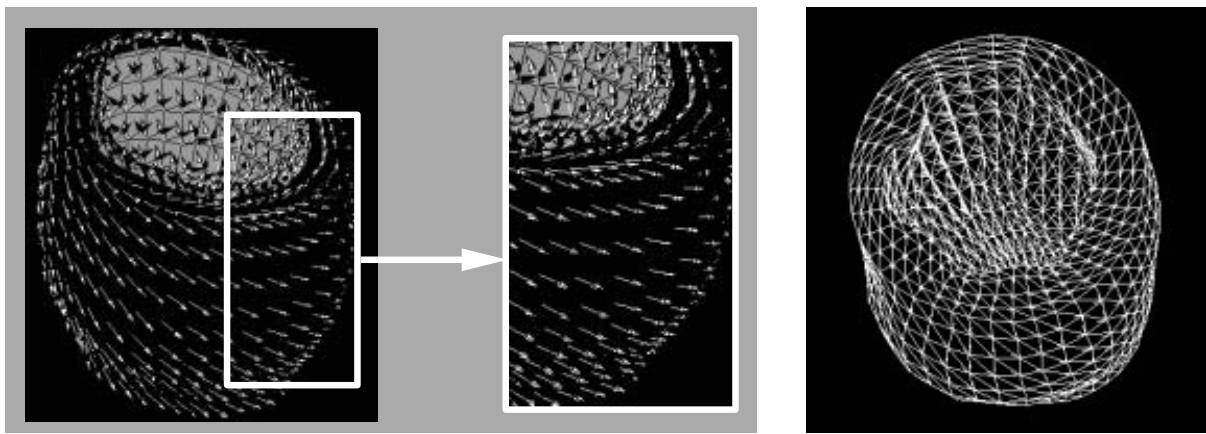

Fig. 1. Left: Fiber direction in the left ventricle as defined in Guccione[5]. Right: Volumetric Model of the LV consisting of hexahedral elements.

The left ventricle of the heart is specifically modeled as a transversely elastic material to account for the preferential stiffness in the fiber direction, using the following material matrix $C$ : 


$$
C^{-1}=\left[\begin{array}{cccccc}
\frac{1}{E_{p}} & \frac{-\nu_{p}}{E_{p}} & \frac{-\nu_{f p}}{E_{f}} & 0 & 0 & 0 \\
\frac{-\nu_{p}}{E_{p}} & \frac{1}{E_{p}} & \frac{-\nu_{f p}}{E_{f}} & 0 & 0 & 0 \\
\frac{-\nu_{f p} E_{f}}{E_{p}} & \frac{-\nu_{f p} E_{f}}{E_{p}} & \frac{1}{E_{f}} & 0 & 0 & 0 \\
0 & 0 & 0 & \frac{2\left(1+\nu_{p}\right)}{E_{p}} & 0 & 0 \\
0 & 0 & 0 & 0 & \frac{1}{G_{f}} & 0 \\
0 & 0 & 0 & 0 & 0 & \frac{1}{G_{f}}
\end{array}\right]
$$

where $E_{f}$ is the fiber stiffness, $E_{p}$ is cross-fiber stiffness and $\nu_{f p}, \nu_{p}$ are the corresponding Poisson's ratios and $G_{f}$ is the shear modulus across fibers. $\left(G_{f} \approx\right.$ $E_{f} /\left(2\left(1+\nu_{f p}\right)\right)$. The fiber stiffness was set to be 3.5 times greater than the crossfiber stiffness [5]. The Poisson's ratios were both set to 0.4 to model approximate incompressibility. Using a Markov Random Field analogy, we can describe the model probabilistically using an equivalent prior probability density function $p(u)$ of the Gibbs form:

$$
p(u)=k_{1} \exp (-W(C, u))
$$

\subsection{Integrating Model and Data}

Having defined both the data term (equation 1) and the model term (equation 4) as probability density functions we naturally proceed to write the overall problem in a Bayesian estimation framework. Given a set of noisy input displacement vectors $u^{m}$, the associated noise model $p\left(u^{m} \mid u\right)$ (data term) and a prior probability density function $p(u)$ (model term), find the best output displacements $\hat{u}$ which maximize the posterior probability $p\left(u \mid u^{m}\right)$. Using Bayes' rule we can write:

$$
\hat{u}=\underset{u}{\arg \max } p\left(u \mid u^{m}\right)=\underset{u}{\arg \max }\left(\frac{p\left(u^{m} \mid u\right) p(u)}{p\left(u^{m}\right)}\right)
$$

Taking logarithms in equation (5) and differentiating with respect to the displacement field $u$ results in a system of partial differential equations, which we solve using the finite element method [3]. To achieve this, a volumetric model of the LV is constructed using hexahedral elements as shown in figure 1. For each frame between end-systole (ES) and end-diastole (ED), a two step problem is posed: (i) solving equation (5) normally and (ii) adjusting the position of all points on the endocardial and epicardial surfaces so they lie on the endocardial and epicardial surfaces at the next frame using a modified nearest-neighbor technique and solving equation (5) once more using this added constraint. This ensures that there is a reduction in the bias in the strain estimates.

\section{Experimental Results}

In this section we present validation of the image derived strains using implanted markers and sonomicrometers as gold standards. 


\subsection{Implanted Image-Opaque Markers:}

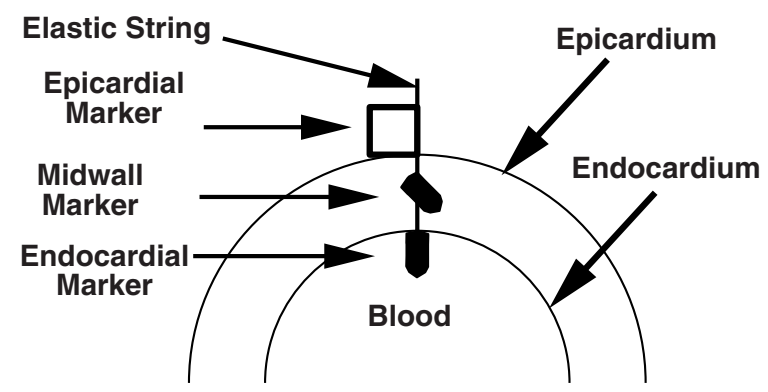

Fig. 2. Implantation of Image-Opaque Markers. This figure shows the arrangement of markers on the myocardium. First a small bullet-shaped copper bead attached to an elastic string was inserted into the blood pool through a needle track. Then the epicardial marker was sutured (stitched) to the myocardium and tied to the elastic string. Finally, the midwall marker was inserted obliquely through a second needle track to a position approximately half-way between the other two markers.

Methodology: To validate the image-derived strains, markers were implanted on canine hearts as follows: First the canine heart was exposed through a thoracotomy. Arrays of endocardial, midwall and epicardial pairs of markers were then implanted as shown in figure 2. They were loosely tethered, combinations of small copper beads (which show up dark in the MR images) at the endocardial wall and the midwall region and small plastic capsules filled with a 200:1 mixture of water to Gd-DTPA at the epicardial wall (which show up bright in the MR images). Marker arrays were placed in two locations on the canine heart wall. The location of each implanted marker is determined in each temporal frame by first manually identifying all pixels which belong to the marker area (as the marker 'image' extends to more than one voxel) and then computing the 3D centroid of that cluster of points, weighted by the grey level ${ }^{1}$. figure 3 shows a short-axis MR slice of the heart with the identified marker pixels shown in blue (left). The marker centroids are shown on the right.

Results: The image-derived strains, estimated using the algorithm described in section 2, were compared to strains derived from implanted markers ${ }^{2}$. In the case of the markers the strains were computed as follows using only the epicardial and

\footnotetext{
${ }^{1}$ In the case of dark markers the image is first inverted.

${ }^{2}$ Note that in the estimation of the image-derived strains, we do not use any information regarding the position of the implanted markers.
} 


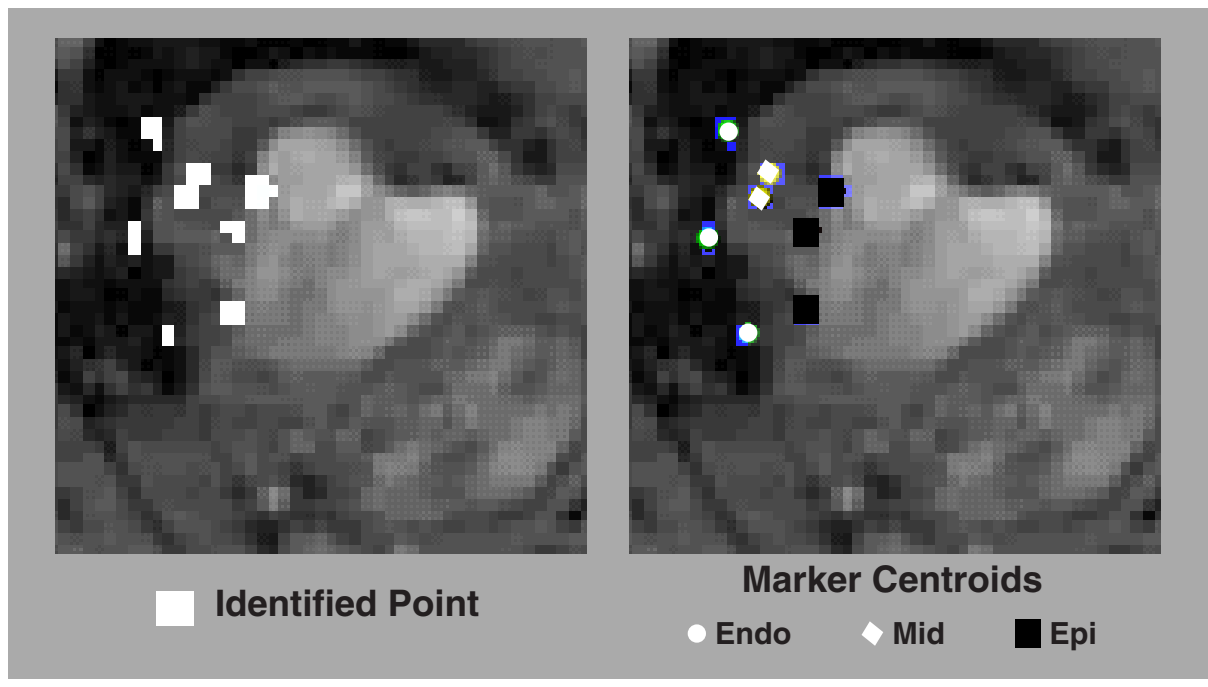

Fig. 3. Localization of implanted markers. Arrays consisting of 12 markers each were placed at two positions on the left ventricle. In this figure, we show the portion of one marker array as it intersected a short-axis MR image slice. A human observer identified the pixels corresponding to each marker (left) and the marker positions (right) were found by calculating centroids of these points.
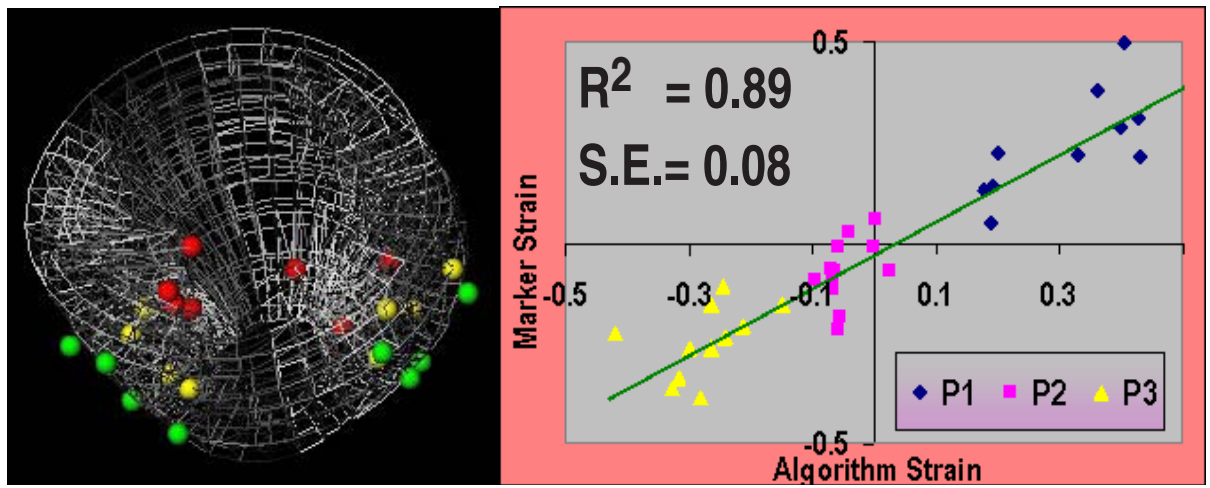

Fig. 4. Algorithm-derived Strains vs. Implanted Marker-derived Strains. Left: Reconstructed LV volume from cine-MRI at ED with marker positions noted as spheres. Right: Scatter plot of principal strains derived from baseline and post-infarction cine-MRI studies using algorithm vs. same strains derived from implanted marker clusters at two positions in the LV wall for $N=4 \operatorname{dogs}$ (There was a total of 12 usable extracted marker arrays). 
endocardial markers. In each region of the LV that contained markers, groups of either 6 or 8 markers (depending on the geometry) were connected to form wedge or hexahedral elements. Given the known displacements, we then calculated the strains between these markers. These strains were compared to the average strains in the elements contained within each marker array. We used principal strains, (these are the eigenvalues of the strain tensor[9]), as the marker arrays where large and included elements where the cardiac-specific directions varied widely.

Comparison results are shown in figure 4 for $N=4$ dogs (2 acquisitions per dog, one pre-occlusion and one post-occlusion). We observe a strong correlation of the principal strain values $\left(r^{2}=0.89\right)$.

\section{$3.2 \quad$ Sonomicrometers}

Methodology: In the case of the 3DE images we validate the strain estimates using implanted sonomicrometers (Sonometrics Corporation, London Ontario, Canada.) The canine heart is again first exposed through a thoracotomy. With the aid of an implantation device constructed in our laboratory, two crystalarrays each consisting of 12 crystals ( 3 sub-epicardial, $\sim 2.0 \mathrm{~mm}, 6$ mid-wall and 3 sub-endocardial, $\sim 0.75 \mathrm{~mm}$ diameter) were placed in the heart wall. Finally, to define a fixed coordinate space, 3 crystals attached to a plexi-glass frame were secured in the pericardial space under the right ventricle.

Digital sonomicrometry employs the time of flight principal of ultrasound to measure the distance between a transmitter and a receiver. A total of 27 crystals are used in each study. The distances between all possible pairs of crystals are recorded along with LV and aortic pressure at a sampling frequency of greater than $125 \mathrm{~Hz}$. There are a number of preprocessing steps involved in obtaining the positions of the crystals over time from the crystal to crystal pair lengths. These are described by Dione[4] (see also Ratcliffe[15].) The efficacy of this technique was illustrated by additional work [10] that showed that the distances obtained with sonomicrometers compared favorably $(r=0.992)$ with those obtained using the more established technique of tracking implanted bead displacements using biplane radiography.

Results: We compared our image-derived strains, estimated using the algorithm described in section 2, to concurrently-estimated strains derived from sonomicrometers at several positions in the LV myocardium in the same dogs. The sonomicrometers were visually located from the images and the two nearest sectors [12] of algorithm-derived strains were selected for comparison purposes. The comparison of the principal strain components in two separate regions for a set of 3 studies showed a strong correlation $\left(r^{2}=0.80\right)$. Here we again compare the principal strains as in the last section. A scatter plot of algorithm-derived principal strains versus sonomicrometer-derived principal strains is shown in figure 5 . 


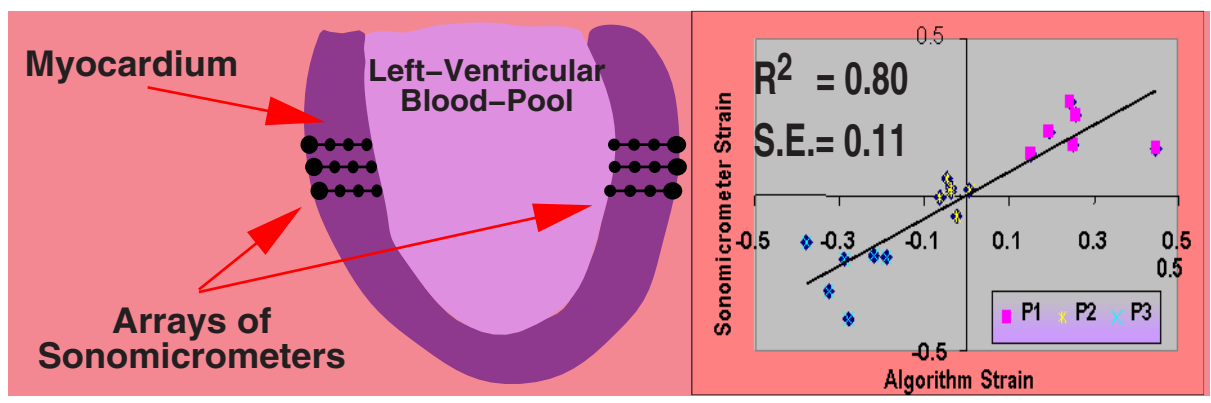

Fig. 5. 3DE Algorithm-Derived Strains vs. Sonomicrometer-derived Strains. Left: Placement of arrays of sonomicrometers in the Left Ventricular Wall (the crystals are shown enlarged, they are barely visible in the images.) Right: Scatter plot of principal strains derived from these 3DE studies using the algorithm vs. same strains derived from sonomicrometer arrays (12 crystals in each cluster) at two positions in the left ventricular wall. Note the high correlation between the two sets of strain values $\left(r^{2}=0.80\right)$.

\section{Conclusions}

In this work, we describe methodology to validate 3D image-derived cardiac deformation estimates, and we use it to compare the output of our algorithm[12] to implanted markers and sonomicrometers. We demonstrate good correlation of the image-derived estimates of strain, from both cine-MRI and 3D Echocardiography, to strains estimated from implanted markers and sonomicrometers, used as gold standards. At this point we only compare transmurally averaged principal strains between end-diastole and end-systole. In the future we hope to extend the validation to account for more time points and also to non-transmurally averaged cardiac specific strains.

\section{References}

[1] A. A. Amini, Y. Chen, R. W. Curwen, V. Manu, and J. Sun. Coupled B-snake grides and constrained thin-plate splines for analysis of $2 \mathrm{D}$ tissue defomations from tagged MRI. IEEE Transactions on Medical Imaging, 17:3:344-356, June 1998.

[2] T. Arts, W. Hunter, A. Douglas, A. Muijtens, and R. Reneman. Description of the deformation of the left ventricle by a kinematic model. J. Biomechanics, 25(10):1119-1127, 1992.

[3] K. Bathe. Finite Element Procedures in Engineering Analysis. Prentice-Hall, New Jersey, 1982.

[4] D. P. Dione, P. Shi, W. Smith, P. De Man, J. Soares, J. S. Duncan, and A. J. Sinusas. Three-dimensional regional left ventricular deformation from digital sonomicrometry. In 19th Annual International Conference of the IEEE Engineering in Medicine and Biology Society, pages 848-851, Chicago, IL, March 1997. 
[5] J. M. Guccione and A. D. McCulloch. Finite element modeling of ventricular mechanics. In P. J. Hunter, A. McCulloch, and P. Nielsen, editors, Theory of Heart, pages 122-144. Springer-Verlag, Berlin, 1991.

[6] E. Haber, D. N. Metaxas, and L. Axel. Motion analysis of the right ventricle from MRI images. In Medical Image Computing and Computer Aided Intervention (MICCAI), pages 177-188, Cambridge, MA, October 1998.

[7] W. S. Kerwin and J. L. Prince. Cardiac material markers from tagged MR images. Medical Image Analysis, 2(4):339-353, 1998.

[8] D. L. Kraitchman, A. A. Young, C. Chang, and L. Axel. Semi-automatic tracking of myocardial motion in MR tagged images. IEEE Transactions on Medical Imaging, 14(3):422-433, September 1995.

[9] L. E. Malvern. Introduction to the Mechanics of a Continuous Medium. PrenticeHall, Englewood Cliffs, New Jersey, 1969.

[10] D. Meoli, R. Mazhari, D. P. Dione, J. Omens, A. McCulloch, and A. J. Sinusas. Three dimensional digital sonomicrometry: Comparison with biplane radiography. In Proceedings of IEEE 24th Annual Northeast Bioengineering Conference, pages 64-67, 1998.

[11] X. Papademetris, J. V. Rambo, D. P. Dione, A. J. Sinusas, and J. S. Duncan. Visually interactive cine-3D segmentation of cardiac MR images. Suppl. to the J. Am. Coll. of Cardiology, 31(2. Suppl. A), February 1998.

[12] X. Papademetris, A. J. Sinusas, D. P. Dione, and J. S. Duncan. 3D cardiac deformation from ultrasound images. In Medical Image Computing and Computer Aided Intervention (MICCAI), pages 420-429, Cambridge, England, September 1999.

[13] X. Papademetris, A. J. Sinusas, D. P. Dione, and J. S. Duncan. Estimation 3D left ventricular deformation from echocardiography. Medical Image Analysis, in-press.

[14] J. L. Prince and E. R. McVeigh. Motion estimation from tagged MR image sequences. IEEE Transactions on Medical Imaging, 11:238-249, June 1992.

[15] M.B. Ratcliffe, K.B. Gupta, J.T. Streicher, E.B. Savage, D.K. Bogen, and L.H. Edmunds. Use of sonomicrometry and multidimensional scaling to determine the three dimensional coordinates of multiple cardiac locations: feasibility and initial implementation. IEEE Trans Biomed Eng, 42:587-598, 1995.

[16] P. Shi, A. J. Sinusas, R. T. Constable, and J. S. Duncan. Volumetric deformation analysis using mechanics-based data fusion: Applications in cardiac motion recovery. International Journal of Computer Vision, 35(1):65-85, November 1999.

[17] P. Shi, A. J. Sinusas, R. T. Constable, E. Ritman, and J. S. Duncan. Point-tracked quantitative analysis of left ventricular motion from $3 \mathrm{D}$ image sequences. IEEE Transactions on Medical Imaging, 19(1):36-50, January 2000.

[18] E. Waks, J. L. Prince, and A. Douglas. Cardiac motion simulator for tagged MRI. In Mathematical Methods in Biomedical Imaging Analysis, pages 182-191, 1996.

[19] A. A. Young, L. Axel, and et al. Validation of tagging with MR imaging to estimate material deformation. Radiology, 188:101-108, 1993. 